\title{
Maturing is part of life
}

DOI:

10.1038/nrm1972

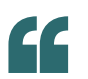

...these two

papers have

shown that the

Golgi cisternae

of $S$. cerevisiae

mature over

time.
When we look in the mirror, we might wish that we could stop time, but - for better or for worse - maturing is part of life. And this seems to be true even at the level of membrane trafficking in cells. For some time, the nature of Golgi cisternae has been debated. Are they stable compartments that retain a characteristic set of Golgi-resident proteins, or are they transient structures that form de novo, mature and then disperse? Two groups - Glick and colleagues and Nakano and coworkers - now answer this question in Nature.

Both groups used three-dimensional time-lapse fluorescencemicroscopy techniques to visualize

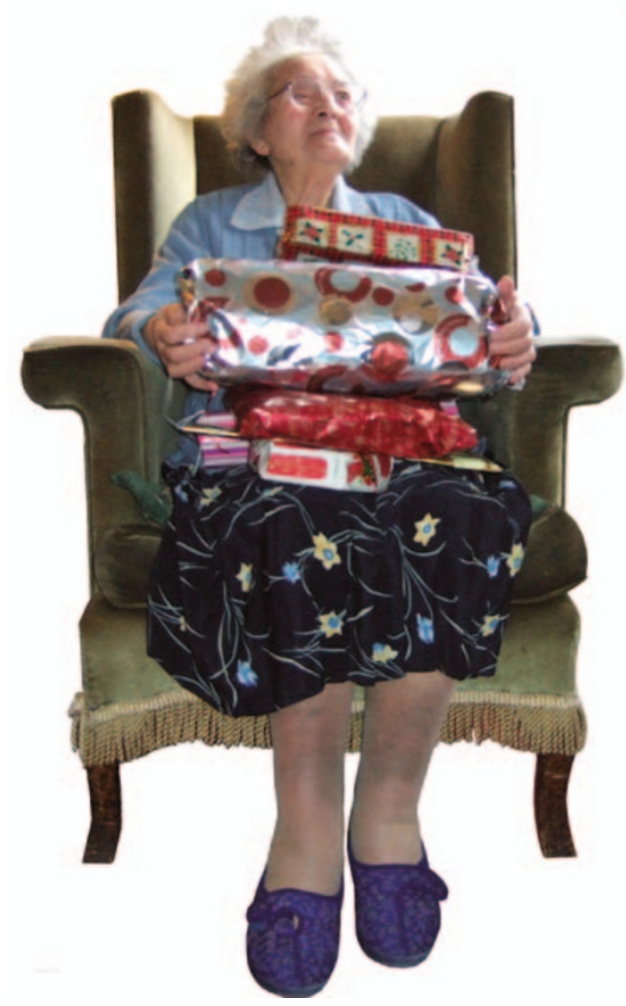

the Golgi apparatus of Saccharomyces cerevisiae (its cisternae are not stacked and can therefore be resolved using such techniques). Furthermore, they both generated fluorescently tagged markers of the different Golgi cisternae, which localized correctly at high expression levels without significantly affecting organelle morphology.

By using several markers of different Golgi cisternae, Glick's group showed that multiple classes of Golgi protein label an individual cisterna only transiently, which is consistent with the cisternal maturation model. They therefore attempted to visualize cisternal maturation by simultaneously tagging the early and late Golgi with green and red fluorescent markers, respectively. Again, consistent with the maturation model, cisternae exhibited green fluorescence for 2-3 minutes, entered a transition phase during which both colours were visible, showed red fluorescence for 2-3 minutes, and then lost detectable fluorescence. Red-to-green colour transitions were never observed.

Nakano's group carried out similar experiments and the colour changes they observed also indicated that the Golgi cisternae of $S$. cerevisiae change their distribution of resident membrane proteins from cis- to trans-resident proteins over time (the colour changes were always unidirectional). They therefore investigated how these membrane components might be transferred between cisternae. Coatomer protein complex-I (COPI)-coated vesicles have been proposed to carry Golgiresident proteins from later to earlier cisternae, and the authors found that a defect in COPI slowed, but did not block, the colour conversion of cisternae. COPI vesicles are therefore important for cisternae conversion, but other mechanisms are probably also involved.

It has been proposed that cisternal maturation might occur too slowly to account for the secretion of some cargo proteins. Glick and colleagues therefore used radioactive pulse-chase approaches to analyse the transport of two secretory cargo proteins (one of which is rapidly secreted in S. cerevisiae). In both cases, they showed that the observed rate of cisternal maturation could account for the kinetics of secretorycargo-protein transport.

In summary, these two papers have shown that the Golgi cisternae of S. cerevisiae mature over time. A challenge for the future will therefore be to elucidate the mechanism that underlies this unidirectional cisternal maturation. Furthermore, the highly dynamic mixing and resegregation of resident proteins in a single cisterna that was observed by Nakano's group has raised many new questions that require further investigation.

\section{Rachel Smallridge}

ORIGINAL RESEARCH PAPERS Losev, E. et al. Golgi maturation visualized in living yeast. Nature 14 May 2006 (doi:10.1038/nature04717)|

Matsuura-Tokita, K. et al. Live imaging of yeast Golgi cisternal maturation. Nature 14 May 2006 (doi:10.1038/nature04737) WEB SITES

Benjamin Glick's laboratory: https:// departmentinfo.uchicago.edu/faculty. phtml?faculty_id $=66$

Akihiko Nakano's laboratory: http://www.riken go.jp/engn/r-world/research/lab/wako/ membrane/index.html 